\title{
The characteristics and needs of older forensic psychiatric patients: A cross-sectional study in secure units within one UK regional service
}

Claudio Di Lorito' Division of Rehabilitation, Ageing and Wellbeing, School of Medicine, University of Nottingham, Nottingham, United Kingdom. ORCID: 0000-0002-8953-0117

Birgit Völlm, Klinik und Poliklinik für Forensische Psychiatrie, Universitätsmedizin Rostock, Rostock, Deutschland. ORCID: 0000-0003-4571-3410

Tom Dening, Division of Psychiatry and Applied Psychology, School of Medicine, University of Nottingham, Nottingham, United Kingdom. ORCID: 0000-0003-3387-4241

Corresponding author: Claudio Di Lorito BA Hons, MSc

Room B119, Division of Rehabilitation and Ageing, School of Medicine, Queen's Medical centre, Nottingham UK, NG7 2UV, United Kingdom

Email: claudio.dilorito1@nottingham.ac.uk

Data availability statement: In dissemination materials no personal identifiable data of the participants were used. Each participant was assigned an anonymised study identity code, and this was used in all research documents and outputs. Access to participants' data is restricted to the research team, the sponsor's designee and relevant regulatory authorities. Anonymised research documents are available upon request from: claudio.dilorito1@ nottingham.ac.uk 


\begin{abstract}
Although the number of older patients in forensic psychiatric settings is increasing, there is limited information around their sociodemographic characteristics, needs and cognitive abilities. This cross-sectional study focuses on patients aged $\geq 50$ years in three (high, medium and low) secure forensic psychiatric services in England. The study comprises: 1. Analysis of sociodemographic characteristics of all patients $(n=94)$ in the services; 2. Analysis of the clinical notes and administration of needs and cognitive assessments to a subsample of patients $(n=41)$. The main outcomes include: sociodemographic characteristics, data on residency, risk, violence, mental and physical health, cognitive ability and individual needs. Data analysis is carried out through descriptive tests and correlation and inferential analyses of outcomes. Results evidence that most patients are White-British single males aged 50-54 years and $88 \%$ have at least one physical health condition. A quarter of the patients has cognitive impairment. The most common psychiatric disorder is Personality Disorder (60\%); comorbid psychiatric disorders are prevalent (54\%). Length of stay averages $6+$ years and is longest in high security. Patients' needs are mostly met. The least met needs are social opportunities. Future comparative research against younger populations could give better context to research findings from this study.
\end{abstract}

Declarations of interest: none

Key words: Forensic psychiatry, older adults, secure services, special needs, cognitive functioning 


\section{Introduction}

Forensic mental health services provide treatment to patients who present an imminent risk to themselves and others (Di Lorito \& Völlm, 2018). Practices vary across countries, owing to different legal systems, regulations and resources (Salize \& Dressing, 2007), but common elements include the security and limitation of freedom imposed to the patients and the provision of medical, psychological and social interventions (Tapp, Warren, Fife-Schaw, Perkins, \& Moore, 2016).

In most western countries the number of older people in forensic settings (e.g. forensic psychiatric hospitals and prisons) has increased in the last decade due to an ageing population, a tougher approach against crime and the prosecution of historical offences (Moll, 2013). A study by Wong, Lumsden, Fenton, \& Fenwick from 1995 found that $8 \%$ of the patients in one UK high security hospital were > 60 years. In 2017, patients > 50 years represented $21 \%$ of the population in another UK high security hospital (Data provided by the Nottinghamshire Healthcare Trust Applied Information Team). A similar trend has been reported in Italy (Di Lorito et al., 2017) and Germany (Di Lorito \& Völlm, 2018).

The increasing number of older patients has been accompanied by a neglect in research (Das, Murray, Driscoll, \& Nimmagadda, 2012). Although extensive literature has reported around older offenders in prison (Hayes, Burns, Turnbull, \& Shaw, 2012) and the community (Needham-Bennett, Parrott, \& Macdonald, 1996), a literature review only found seven papers on older forensic psychiatric patients (Di Lorito, Völlm, \& Dening, 2018). A unique clinical picture emerges from these studies. Older patients present with high prevalence of psychotic illness (91.6\% in Yorston and Taylor, 2009). Personality disorder affects 3\% (Curtice, Parker, Wismayer, \& Tomison, 2003) to $16.6 \%$ (Yorston \& Taylor, 2009) of the older patients, and 
depression between 6\% (Curtice, Parker, Wismayer, \& Tomison, 2003) and 42\% (lifetime prevalence) (Coid, Fazel, \& Kahtan, 2002)

Older patients also have frequent physical health conditions, such as heart disease and hypertension, and dementia (Coid, Fazel, \& Kahtan, 2002; Shah, 2006), which may require highly specialised care. It is unclear, however, whether forensic psychiatric services are currently meeting the complex needs of older patients. It is therefore timely to conduct further research.

This study focused on three in-patient forensic psychiatric settings in England at different levels of security: Rampton Hospital (high security) (HS), Arnold Lodge (medium security) (MS) and the Wells Road Centre (low security) (LS), all part of Nottinghamshire Healthcare National Health Service Foundation Trust.

It had two objectives:

(i). To investigate the general demographic characteristics of all the older forensic psychiatric patients in the three services;

(ii). To gather in-depth data on a sample of patients in the three services, including:

- Socio-demographic data

- Information about their residency / admission / offences

- The type of treatment they receive

- Their history of violent incidents during admission

- Risk

- Mental and physical health

- Their individual needs and whether these are met / unmet

- Their cognitive abilities. 


\section{Methods}

This study is reported according to the principles of the STROBE Statement (Von Elm et al., 2014). It is part of a larger project entitled 'The characteristics, needs and individual experience of older forensic psychiatric patients: A cross-sectional study in three secure units within one regional service', which also comprised two qualitative studies previously published in this journal (Di Lorito, Dening, \& Völlm, 2019; Di Lorito, Dening, \& Völlm, 2018).

\section{Participants}

For objective 1 , we selected all patients aged $\geq 50$ years in the three services. Data were provided in anonymised form by the Trust, so consent from the patients was not required.

For objective 2, the patients signed a consent form, agreeing on having their data used for the purposed of this research. In compliance with the UK Data Protection Act 1998, each patient was assigned an anonymised identification code for use on research documents, which were treated as confidential and stored in a secure office, accessible only to the investigators and the regulatory authorities.

Patients for objective 2 met the following inclusion criteria:

1. Aged $\geq 50$ years. We acknowledge that ageing is a subjective experience and that the trend in psychiatry is to move away from chronological age in determining appropriateness of service. However, research requires an arbitrary age criterion. Regrettably, the existing studies in forensic psychiatry have adopted different age cutoffs, from 55 (Lightbody, Gow, \& Gibb, 2010) to 65 years old (Curtice, Parker, Wismayer, \& Tomison, 2003; Tomar, Treasden \& Shah, 2006). 
We turned our attention to the prison literature, as the prison system is the main source of admission in secure care for older forensic psychiatric patients (Curtice, Parker, Wismayer, \& Tomison, 2003; Coid, Fazel, \& Kahtan, 2002; Lightbody, Gow, $\&$ Gibb, 2010). The existing studies agree on an age cut-off of 50 years old. We therefore adopted this inclusion criterion.

2. Currently resident in one of the three services.

3. Able to give consent.

4. Deemed fit to participate by the clinical team and the researcher.

\section{Data collection}

\section{Objective 1}

Data on age, gender, nationality, ethnicity, marital status and admission date of all the patients $\geq 50$ years staying in the three services were gathered on 10 October 2017 . The data were provided to the first author by the Trust.

\section{Objective 2}

Data for objective 2 were collected between May and December 2017 by the first author (CDL). Our detailed assessment of individual patients had three components:

1. Examination of clinical records, to obtain data on: (i) Socio-demographics; (ii) residency, admission and offences; (iii) mental health and treatment; (iv) psychopathy score [assessed though the Psychopathy Check List - Revised (PCL-R) (Hare, 1991); (v) physical health; and (vi) risk [assessed through the Historical Clinical Risk Management-20, Version 3 (HCR-20 V3) (Douglas, Hart, Webster, \& Belfrage, 2013) 
and incidents / seclusion. We chose to collect these data, as they are available for all patients on the Trust's electronic file system. The first author (CDL) received raining and obtained permission from the regulatory authority to access patients' files.

2. Assessment of individual needs through the Camberwell Assessment Needs Forensic version - Short (CANFOR-S) (Thomas et al., 2003). The CANFOR-S includes 25 items, investigating needs in key areas of daily living (e.g. accommodation, social life, treatment) (Stuart et al., 2008). Validation studies have found high inter-rater reliability across service users (0.991) and staff (0.998) and test-retest reliability at two weeks' time intervals (Stuart et al., 2008).

We added to the original assessment four items from the Camberwell Assessment Needs for the elderly - Short (CANE-S) (Orrell \& Hancock, 2004) and the Camberwell Assessment Needs for Intellectual Disabilities - Short (CANDID-S) (Xenitidis, Slade, Thornicroft, \& Bouras, 2000) that were relevant to our target group: eyesight/hearing, mobility, abuse/neglect, and incontinence. The first author (CDL) received training through the CANFOR-S manual before administering the assessment.

3. Assessment of cognition through the Cambridge Cognitive Examination (CAMCOGR) (Roth, Huppert, Mountjoy, \& Tym, 1998), a 50-item scale investigating orientation, language, memory, attention, praxis, calculation, abstraction, and perception. The CAMCOG-R has been validated and widely utilised in international studies (Hodges, 2007). It is very sensitive to detect mild cognitive impairment and presents low ceiling effects (Lindeboom, Ter Horst, Hooyer, Dinkgreve, \& Jonker, 
1993). The first author received training on the use of the CAMCOG-R through the

CFAS (Cognitive Function and Ageing Studies) at the University of Cambridge.

\section{Data analysis}

Data were analysed through SPSS 22 (IBM, 2013). In addition to descriptive tests of all variables, we undertook several correlation and parametric/non-parametric inferential analyses (Table 1).

When carrying out further data analysis associating age with length of stay, psychiatric diagnosis, physical health conditions and needs, we further divided our sample into two subgroups: $<55$ years old $(n=20)$ and $\geq 55$ years old $(n=21)$, to test whether among older patients, there were any differences between the more and less senior ones.

We also conducted the following comparison analyses:

- In relation to risk, we compared HCR-20 V3 scores published in a study on 532 patients staying in Rampton Hospital (one of our research sites) over five years (Morrissey, Beeley, \& Milton, 2014) against the scores obtained by our patients. The study reported data by diagnostic ward category, so we compared these ratings against two sub-samples of our patients (i.e. HS and MS), by using the same categorisation.

- In relation to CAMCOG-R score, we compared our results against data from a large community study on 730 older adults (Pereiro, Ramos-Lema, Juncos-Rabadán, Facal, \& Lojo-Seoane, 2015). In this study, cognitive performance was reported by age subsamples (i.e. $<60$ and $\geq 60$ years old), so we divided our sample into the same age categories. 


\section{Ethical approval}

The study received ethics approval (REC reference: 16/EM/0505) through the Health Research Authority (HRA). The Nottinghamshire Healthcare NHS Foundation Trust Research \& Development (R\&D) Department gave permission to run the study in the three services and use anonymised patients' data.

\section{Results}

\section{Objective 1}

The number of patients aged $\geq 50$ years residing in the three services was 94 . They represented $18.9 \%$ of a total number of 496 patients. Patients $\geq 50$ years represented $21 \%$ of the population in HS, $15.5 \%$ in MS and $14.4 \%$ in LS. Patients' age ranged from 50 to 73 years old (mean $=55.6, \mathrm{SD} \pm 5.0)$. No statistically significant association was found between age and security of service ( $p>0.05)$.

There were ten female patients (10.6\%), with a female to male ratio of 1:8. Seven female patients were in HS, two in MS and one in LS. The patients had stayed in the service an average of 8 years and 6 months since their admission to the current service. Patients in HS had a statistically significant longer stay than those in MS or LS (Kruskal-Wallis $\mathrm{H}=36.4$, $\mathrm{p}$ $<0.001)$. No statistically significant difference was found between medium and low security. Female patients had stayed in the service for significantly shorter periods than male patients (mean $=$ five years and three months versus eight years and nine months; Mann-Whitney $\mathrm{U}=$ 284; $\mathrm{p}=0.049$ ). Sociodemographic details and data on residency are summarised in Table 2.

\section{Objective 2}

Forty-one patients ( $44 \%$ of the total aged $\geq 50$ years) were involved in objective 2 of the study (Figure 1). The sample was representative of the overall population of patients $\geq 50$ 
years in the three services in relation to age $($ sample mean $=55.5$, population mean $=55.6$, gender distribution, ethnicity and marital status, with no statistically significant differences ( $>0.05)$ between the two groups. Sociodemographic details, data on residency and index offence are summarised in Table 3.

\section{Residency, admission and offences}

The mean length of stay in their current admission was six years and three months. Patients in HS had a statistically significant longer stay than those in MS and LS, the length of stay in HS being 2.6 times of that in MS and 4.2 that in LS (Kruskal-Wallis test $H=10.7, \mathrm{p}<0.001$ ). Patients $\geq 55$ years old had a significantly longer stay (six years and two months), compared to patients $<55$ years old (three years and three months) (Mann-Whitney $\mathrm{U}=133 ; \mathrm{p}=0.04$ ). Prison was the main source of admission for HS patients $(n=11 ; 45.8 \%)$; in MS it was high security $(n=4 ; 40 \%)$, and in LS the community $(n=4 ; 57 \%)$. The most frequent Mental Health Act Sections were $37 / 41^{1}(n=17,41.5 \%)$ and $47 / 49^{2}(n=14,34.1 \%)$. In England and Wales, Section 37 is given by Crown Court if the person should be in hospital instead of prison. Section 41 is a restriction order, added to a section 37 if the person is a risk to the public. Section 47/49 is given by the Ministry of Justice to transfer a sentenced prisoner with mental illness to hospital.

Admission history evidenced that twenty-six patients (63\%) had never been admitted to secure forensic psychiatric services before. Previous admission was not significantly associated with age, gender or length of stay.

Nineteen patients (46.3\%) were in the Male Mental Health wards (i.e. male patients with a primary diagnosis of mental illness), 14 (34.1\%) in Personality Disorder (PD) wards (i.e. 
male patients legally classified under the label of Personality Disorder), four (10\%) in the Male LD wards (i.e. male patients with a Learning Disability), three (7\%) in women's services (i.e. all treatment stages and diagnoses) and one (2\%) in male community integration accommodation (i.e. a rehabilitation facility for male patients moving on from low secure inpatient services).

All but one patient had a current index offence (97.6\%), and five patients (12.2\%) had multiple index offences. The most prevalent index offence was assault $(\mathrm{n}=16 ; 39 \%)$. For 27 patients $(65.8 \%)$ there were previous offences recorded. There was no significant association between index offence, gender and age.

Mental health, physical health and treatment

All data are summarised in Table 4 . We found that mood and anxiety disorders were more prevalent among female patients, substance abuse and comorbid conditions among patients < 55 years old, and substance abuse among White non-British patients. Patients taking psychotropic medication were more likely to have a physical health condition $(\mathrm{p}<0.05)$. We also found that obesity was significantly more prevalent among patients $<55(\mathrm{n}=9 ; 47 \%)$ compared to those $\geq 55$ years old $(\mathrm{n}=1 ; 5 \%)$

Risk of violence and incidents / seclusion

The mean score on the HCR-20 V3 (Douglas, Hart, Webster, \& Belfrage, 2013) was 27/40 $(\mathrm{SD}=6.8)$. This is considered a medium risk.

When carrying out our post hoc analysis comparing scores from our HS and MS subsamples with the previous study carried out in HS (Morrissey, Beeley, \& Milton, 2014), patients from MS scored significantly higher in the historical, risk and total score, compared to both our patients in the HS subsample and in the previous study sample, as evidenced by one way 
ANOVAs and Tukey post hoc tests $(F(2,15)=10.7, p=.001) ;(F(2,15)=5.2, p=.01$; $(F(2,15)=6.3, p=.01)$.

Higher HCR-20 V3 score was significantly associated with Personality Disorder, comorbid psychiatric disorders and an index offence of murder $(\mathrm{p}<0.05)$. HCR-20 V3 clinical score was associated with taking benzodiazepines (Mann-Whitney $\mathrm{U}=39.5, \mathrm{p}=0.02$ ).

According to incident report data from the past two years, 29 patients $(70.7 \%)$ had engaged in verbal or physical assault and $11(26.8 \%)$ in self-harm or attempted suicide. All the female patients $(n=3)$ had engaged in self-harm, compared to $21 \%$ of the males $(n=8)$, a statistically significant difference $(\mathrm{p}<0.05)$ Fourteen patients $(34.1 \%)$ had been secluded at least once over the last two years. Seclusion was imposed on 18 (43.5\%) patients in HS, compared to $20 \%-30 \%$ in the other services and was significantly associated with Personality Disorder $(\mathrm{p}<0.05)$.

\section{Camberwell Assessment Needs - Forensic Short version}

The patients reported they had no needs in 10 areas of daily living, on average (SD 3.8). The items scored the most frequently as "no problem experienced in this area" were "arson" $(n=37 ; 90 \%)$, "safety to self" ( $n=35 ; 86 \%)$, "safety to others" ( $n=33 ; 81 \%)$, "alcohol” (n=33; $81 \%)$, and "sexual offences" ( $n=32 ; 78 \%)$. There was a significantly higher number of needs for female patients, patients with Learning Disability, and patients above 55 years old $(\mathrm{p}<0.05)$.

The patients reported their needs were met needs in 12 areas of daily living, on average (SD 3.5). Needs were most met in relation to "Eyesight/hearing/communication" ( $\mathrm{n}=32 ; 78 \%)$; "Treatment" ( $\mathrm{n}=30 ; 74 \%)$, "Information about condition/treatment" $(\mathrm{n}=29 ; 71 \%)$, "Food" and "Money" (n=28; 68\%). There were significantly more met needs for female patients, patients 
with Learning Disability and patients with comorbid conditions, as evidenced through several independent samples t-tests $(\mathrm{p}<0.05)$.

The number of met needs was significantly higher in the Learning Disability, compared to the Male Mental Health and the Male Personality Disorder wards, as evidenced by a One-way ANOVA and post hoc Tukey test $(F(3,36)=4.95, p=0.006)$. No differences were found between other wards ( $p>0.05)$. A statistically significant association between multiple I assume this is multiple modalities of treatment (as listed in Table 4) and met needs was evidenced through an Independent-sample T-test $(\mathrm{p}<0.05)$.

Patients reported their needs were unmet in 4 areas of daily living, on average (SD 3.0). The need was most unmet for "Company" (n=16; 39\%), "Telephone" $(\mathrm{n}=15 ; 37 \%)$, "Sexual expression" and "Daytime activities" (n=14; 34\%). Patients below 55 years old had more unmet needs than those above, as evidenced through a Mann-Whitney test ( $U=118 ; \mathrm{p}=0.01)$. The overall ratio of met to unmet needs was 3:1.

\section{Cognitive Assessment}

Data on cognition was heavily affected by the very low score of three respondents with a Learning Disability (LD) in HS. When the three outliers were excluded, the mean CAMCOG score was 86/100 (SD = 16; Range 20-98). Eight patients (21\%) scored below the conventional cut-off of 80 points for cognitive impairment. Of these, three were in HS (37\%), and five (63\%) in MS; six (75\%) were males and two (25\%) females. When carrying out a post hoc analysis comparing the forensic sample against the community sample (Pereiro, Ramos-Lema, Juncos-Rabadán, Facal, \& Lojo-Seoane, 2015), the CAMCOG-R scores were not statistically significantly different.

No significant association was found between CAMCOG performance and gender, length of stay, age and type of psychotropic medication. A moderate statistically significant negative 
correlation $\left(r_{s}=-.40, p=0.01\right)$ was found with total HCR-20 V3 score, meaning higher CAMCOG score was associated with lower risk. Patients with higher PCL-R scores, Personality Disorder, Learning Disability, mood disorders, and comorbid psychiatric conditions also had statistically significantly lower CAMCOG scores $(\mathrm{p}<0.05)$. In addition, a higher CAMCOG score was associated with fewer unmet needs $\left(r_{s}=-.38, p=0.02\right)$.

\section{Discussion}

This study presents data on the characteristics, cognition and individual needs of a sample of patients $\geq 50$ years old from three forensic psychiatric services within Nottinghamshire healthcare National Health Service Foundation Trust. We found that most patients were single White British males aged 50-55 years, admitted from prison and with an index offence of assault (i.e. common assault, battery, assault occasioning actual bodily harm or assault occasioning grievous bodily harm).

While the typical (i.e. patients of all ages) length of stay in HS averages four and a half years, and in MS one year and a half (Völlm et al., 2017), our sample had much longer stay in secure care. We also observed a longer stay for patients over 55 years old. These findings raise the question concerning age at time of sentencing (information that was not available to us), which may have an impact on length of stay. Our findings may also support the growing body of evidence around the difficulties for older patients to move along the care pathway in a reasonable amount of time and the risk that they might be kept at unnecessarily higher levels of security (Das, Murray, Driscoll, \& Nimmagadda, 2012).

In line with previous studies (Curtice, Parker, Wismayer, \& Tomison, 2003), the patients presented with frequent physical conditions, adding to the burden of their psychiatric disorders. Obesity affected almost half of the patients aged 50- 55 years, pointing at the importance of implementing effective weight management plans in secure settings. As in 
previous studies (Shah, 2006; Lightbody, Gow, \& Gibb, 2010), we also found an association between medical prescriptions and physical conditions, although the direction of this relationship was unclear. Regardless, this finding confirms the relevance of physical ailments in the context of psychiatric illness in secure care.

Personality disorder (PD) was the most frequent diagnosis, followed by psychotic disorders; the patients typically presented with high comorbid psychiatric conditions. Patients in the Personality Disorder directorate within MS scored statistically significantly higher than those in HS in HCR-20 V3 history, risk and total scores. This may be explained by longer stay, more intensive treatment and higher restrictiveness in HS, which may reduce patients' risk over time. It may also suggest that older patients are kept in high security even though their risk does not require such level of security.

Results from the needs assessment evidenced that most of the patients' needs were met. However, as previously reported (Das, Murray, Driscoll, \& Nimmagadda, 2012), unmet needs were relatively common in the areas of social life. We also found that the patients $<55$ reported a statistically significantly higher number of unmet needs than the patients $>55$. This may reflect that the staff neglect the younger, who are perceived as less vulnerable, or that the older patients raise fewer complaints (and report fewer unmet needs), as found in previous studies (Her Majesty’s Inspectorate of Prisons, 2004).

Cognitive impairment was quite prevalent, experienced by over one fifth of the patients in our sample. Although we did not include four patients who were unable to give consent, cognitive impairment was still higher than reported in prison studies [up to 19\% (Combalbert et al., 2016)] and community studies [up to $6 \%$ when calculating the mean of the values by age group (Rait et al., 2005)]. The nature of cognitive impairment in our sample was not clear. It might be a symptom of dementia or the result of brain injury, medications and 
psychiatric conditions such as schizophrenia and substance dependence (Iverson, Brooks, \& Holdnack, 2008). Although we urge caution in drawing any conclusions, findings from this study highlight how widespread cognitive impairment is among older forensic psychiatric patients, requiring consideration of their unique treatment and care needs.

Our study has certain strengths and limitations. Data collection was based on both clinical data and on patients' views. This is quite novel in the panorama of old age forensic psychiatric research, which has traditionally relied on reports from staff, thus neglecting the view of the primary stakeholder, the patients themselves. Our sample was representative of the overall population of patients $\geq 50$ years in the three services and in line with the mean sample size ( $\mathrm{n}=42)$ of previous studies (Das, Murray, Driscoll, \& Nimmagadda, 2012; Yorston \& Taylor, 2009; Curtice, Parker, Wismayer, \& Tomison, 2003; Coid, Fazel, \& Kahtan, 2002; Shah, 2006; Lightbody, Gow, \& Gibb, 2010; Tomar, Treasaden, \& Shah, 2005; Paradis, Broner, Maher, \& O'rourke, 2000).

Study limitations mostly pertain to the poor generalisability of findings. It is possible that patients with overall better functioning were more likely to agree to take part than those with more severe presentation of symptoms. Also, given the complexity of involving patients with severe cognitive impairment, we excluded the patients who could not give consent. Another limitation, common to studies of older forensic populations, is the small sample size, which limits the capacity to identify subgroup differences (e.g. by gender).

Given the limited capacity of this study and its local scope (within one single Trust in England), it would be ideal for future research to generate more representative data. We also advocate for inclusion of all older patients, because the methods we adopted are not intrusive and it is to the disadvantage of the most vulnerable patients if we do not fully understand their needs. 
Ideally, we would have had a comparison group (i.e. patients $\leq 50$ years), which would better contextualise our findings. It is perhaps of interest to note that only one of the existing old age forensic psychiatric studies included a comparison group (Di Lorito, Völlm, \& Dening, 2018). We acknowledge that we cannot infer from our data to what extent the issues are different for older versus younger patients. However, some of the perspectives of ageing in secure settings are reported in a companion qualitative study with this sample (reference anonymised). In addition, research has traditionally neglected older forensic populations and very little information is available at present. It was therefore our goal to undertake an indepth enquiry and dedicate our full resources to capturing as much comprehensive information as possible around the older patients. In acknowledging this limitation, we also believe that this kind of exploratory, descriptive study is needed as a foundation for future research.

In relation to the potential implications for practice, study findings revealed that the patients experience some barriers to recovery, including poor physical health, cognitive impairment, increased length of stay, risk of social isolation and potential inappropriateness of placement. We argue that these may affect older patients' motivation or ability to effectively engage in treatment. In our study, engagement in multiple therapies was statistically significantly associated with more met needs, which is encouraging. Therefore, age-related barriers of access to treatment should be carefully considered in the context of recovery outcomes. It may be beneficial for secure services to implement more inclusive strategies to ensure equal chances of recovery to the older patients.

Disclaimer: This study is funded by the National Institute for Health Research (NIHR) Collaboration for Leadership in Applied Health Research and Care East Midlands (CLAHRC EM). The views expressed are those of the author(s) and not necessarily those of the NIHR or the Department of Health and Social Care. 


\section{References}

Coid, J., Fazel, S., \& Kahtan, N. (2002). Elderly patients admitted to secure forensic psychiatry services. The Journal of Forensic Psychiatry, 13, 416-427. DOI: $10.1080 / 09585180210154470$

Combalbert, N., Pennequin, V., Ferrand, C., Vandevyvère, R., Armand, M., \& Geffray, B. (2016). Mental disorders and cognitive impairment in ageing offenders, The Journal of Forensic Psychiatry \& Psychology, 27, 853-866, DOI: $10.1080 / 14789949.2016 .1244277$

Curtice, M., Parker, J., Wismayer, F.S., \& Tomison, A. (2003). The elderly offender: an 11year survey of referrals to a regional forensic psychiatric service. The Journal of Forensic Psychiatry \& Psychology, 14, 253-265. DOI: 10.1080/1478994031000077989

Das, K., Murray, K., Driscoll, R., \& Nimmagadda, S. (2012). Assessment of healthcare and placement needs in an older forensic psychiatric population in comparison to a younger forensic psychiatric population: Comment. International Psychogeriatrics, 24, 1188-1190.

Di Lorito, C., Castelletti, L., Tripi, G., Gandellini, M.G., Dening, T., \& Völlm, B. (2017). The individual experience of ageing patients and the current service 1 provision in the context of Italian forensic psychiatry: A case-study. Journal of Forensic Nursing, 13, 118-125.

Di Lorito, C., Dening, T., \& Völlm, B. (2019). Ageing in forensic psychiatric secure settings: the views of members of staff. The Journal of Forensic Psychiatry \& Psychology, 30(2), 270285.

Di Lorito, C., Dening, T., \& Völlm, B. (2018). Ageing in forensic psychiatric secure settings: the voice of older patients. The Journal of Forensic Psychiatry \& Psychology, 29(6), 934960.

Di Lorito, C., \& Völlm B. (2018). Older people as victims and perpetrators of crime. In Dening, T., Thomas, A., Stewart, R., \& Taylor, J-P. (Eds.). Oxford Textbook of Old Age Psychiatry, 3rd Edition. Oxford University Press.

Di Lorito, C., Völlm, B., \& Dening, T. (2018). Ageing patients in forensic psychiatric settings: A review of the literature. International Journal of Geriatric Psychiatry. DOI: $10.1002 /$ gps.4981

Douglas, K. S., Hart, S. D., Webster, C. D., \& Belfrage, H. (2013). HCR-20V3: Assessing risk of violence - User guide. Burnaby, Canada: Mental Health, Law, and Policy Institute, Simon Fraser University.

Hare, R. D. (1991). The Hare Psychopathy Checklist-Revised. (Toronto: Multi-Health Systems)

Hayes, A. J., Burns, A., Turnbull, P. \& Shaw, J. J. (2012). The health and social needs of older male prisoners. International Journal of Geriatric Psychiatry, 27, 1155-1162.

Her Majesty's Inspectorate of Prisons (2004). No problems - old and quiet: Older prisoners in England and Wales - a thematic review. London: HM Inspectorate of Prisons. Accessed 
24.11.17 at: https://www.justiceinspectorates.gov.uk/hmiprisons/wpcontent/uploads/sites/4/2014/08/OlderPrisoners-2004.pdf

Hodges, J.R. (2007). Cognitive Assessment for Clinicians: Second Edition. Oxford: Oxford University Press.

IBM SPSS Statistics for Windows, Version 22.0. Released 2013. Armonk, New York: IBM Corporation.

Iverson, G.L., Brooks, B.L., \& Holdnack, J.A. (2008). Misdiagnosis of cognitive impairment in forensic neuropsychology. Neuropsychology in the courtroom: Expert analysis of reports and testimony, pp.243-266.

Lightbody, E., Gow, R.L., \& Gibb, R. (2010). A survey of older adult patients in special secure psychiatric care in Scotland from 1998 to 2007. The Journal of Forensic Psychiatry \& Psychology, 21, 966-974. DOI: 10.1080/14789949.2010.504858

Lindeboom, J., Ter Horst, R., Hooyer, C., Dinkgreve, M., \& Jonker, C. (1993). Some psychometric properties of the CAMCOG. Psychological Medicine, 23, 213-219.

Moll, A. (2013). Losing track of time: dementia and the ageing prison population. Mental Health Foundation, London. Available at:

http://www.mentalhealth.org.uk/content/assets/PDF/publications/losing-track-of-time2013.pdf?view=Standard [Accessed 10 Oct. 2017]

Morrissey, C., Beeley, C., \& Milton, J. (2014). Longitudinal HCR-20 scores in a high-secure psychiatric hospital. Criminal Behaviour and Mental Health, 24, 169-180.

Needham-Bennett, H., Parrott, J., \& Macdonald, A. J. D. (1996). Psychiatric disorder and policing the elderly offender. Criminal Behaviour and Mental Health, 6, 241-252.

Orrell, M. \& Hancock, G. (2004). CANE: Camberwell Assessment of Need for the Elderly. A Needs Assessment for Older Mental Health Service Users. London: Gaskell.

Paradis, C., Broner, N., Maher, L.M., \& O'rourke, T. (2000). Mentally Ill Elderly Jail Detainees. Journal of Offender Rehabilitation, 31, 77-86. DOI: 10.1300/J076v31n01_05

Pereiro, A. X., Ramos-Lema, S., Juncos-Rabadán, O., Facal D., \& Lojo-Seoane, C. (2015). Normative scores of the Cambridge Cognitive Examination-Revised in healthy Spanish population. Psicothema, 27, 32-39. doi: 10.7334/psicothema2014.169

Rait, G., Fletcher, A., Smeeth, L., Brayne, C., Stirling, S., Nunes, M., ... Tulloch, A.J. (2005). Prevalence of cognitive impairment: Results from the MRC trial of assessment and management of older people in the community. Age and Ageing, 34, 242-248.

Roth, M., Huppert, F.A., Mountjoy, C.Q., \& Tym, E. (1998). CAMDEX-R: The Cambridge Examination for Mental Disorders of the Elderly - Revised. Cambridge: Cambridge University Press.

Salize, H. J., \& Dressing, H. (2007). Admission of mentally disordered offenders to specialized forensic care in fifteen European Union member states. Social psychiatry and psychiatric epidemiology, 42(4), 336-342. 
Shah, A. (2006). An Audit of a Specialist Old Age Psychiatry Liaison Service to a Medium and a High Secure Forensic Psychiatry Unit. Medicine, Science, and the Law, 46, 99-104.

Stuart, D. M. T., Slade, M., McCrone, P., Harty, M.A., Parrott, J., Thornicroft, G., \& Leese, M. (2008). The reliability and validity of the forensic Camberwell Assessment of Need (CANFOR): a needs assessment for forensic mental health service users. International Journal of Methods in Psychiatric Research, 17, 111-120.

Tapp, J., Warren, F., Fife-Schaw, C., Perkins, D., \& Moore, E. (2016). Essential elements of treatment and care in high secure forensic inpatient services: an expert consensus study. Journal of Forensic Practice, 18, 189-203.

Thomas, S.D.M., Harty, M.A., Parrott, J., McCrone, P., Slade, M., \& Thornicroft, G. (2003) CANFOR: Camberwell Assessment of Need - Forensic Version. London: Gaskell.

Tomar, R., Treasaden, I.H., \& Shah, A.K. (2005). Is there a case for a specialist forensic psychiatry service for the elderly? International Journal of Geriatric Psychiatry, 20, 51-56. DOI: $10.1002 / g p s .1247$

Völlm, B., Edworthy, R., Holley, J., Talbot, E., Majid, S., Duggan, C., ... \& McDonald, R. (2017). A mixed-methods study exploring the characteristics and needs of long-stay patients in high and medium secure settings in England: implications for service organisation. Health Services and Delivery Research, 5(11).

Von Elm, E., Altman, D. G., Egger, M., Pocock, S. J., Gøtzsche, P. C., Vandenbroucke, J. P., $\&$ Strobe Initiative. (2014). The Strengthening the Reporting of Observational Studies in Epidemiology (STROBE) Statement: guidelines for reporting observational studies. International journal of surgery, 12(12), 1495-1499.

Wong, M.T.H., Lumsden, J., Fenton, G.W., \& Fenwick, P.B.C. (1995). Elderly offenders in a maximum security mental hospital. Aggressive Behaviour, 21, 321-324.

Xenitidis, K., Slade, M., Thornicroft, G., \& Bouras, N. (2000). CANDID: Camberwell Assessment of Need for Adults with Developmental and Intellectual Disabilities. Royal College of Psychiatrists: London.

Yorston, G., \& Taylor, P.J. (2009). Older patients in an English high security hospital: a qualitative study of the experiences and attitudes of patients aged 60 and over and their care staff in Broadmoor Hospital. The Journal of Forensic Psychiatry \& Psychology, 20, 255-267. DOI: $10.1080 / 14789940802327259$ 
Figure 1. Selection of participants

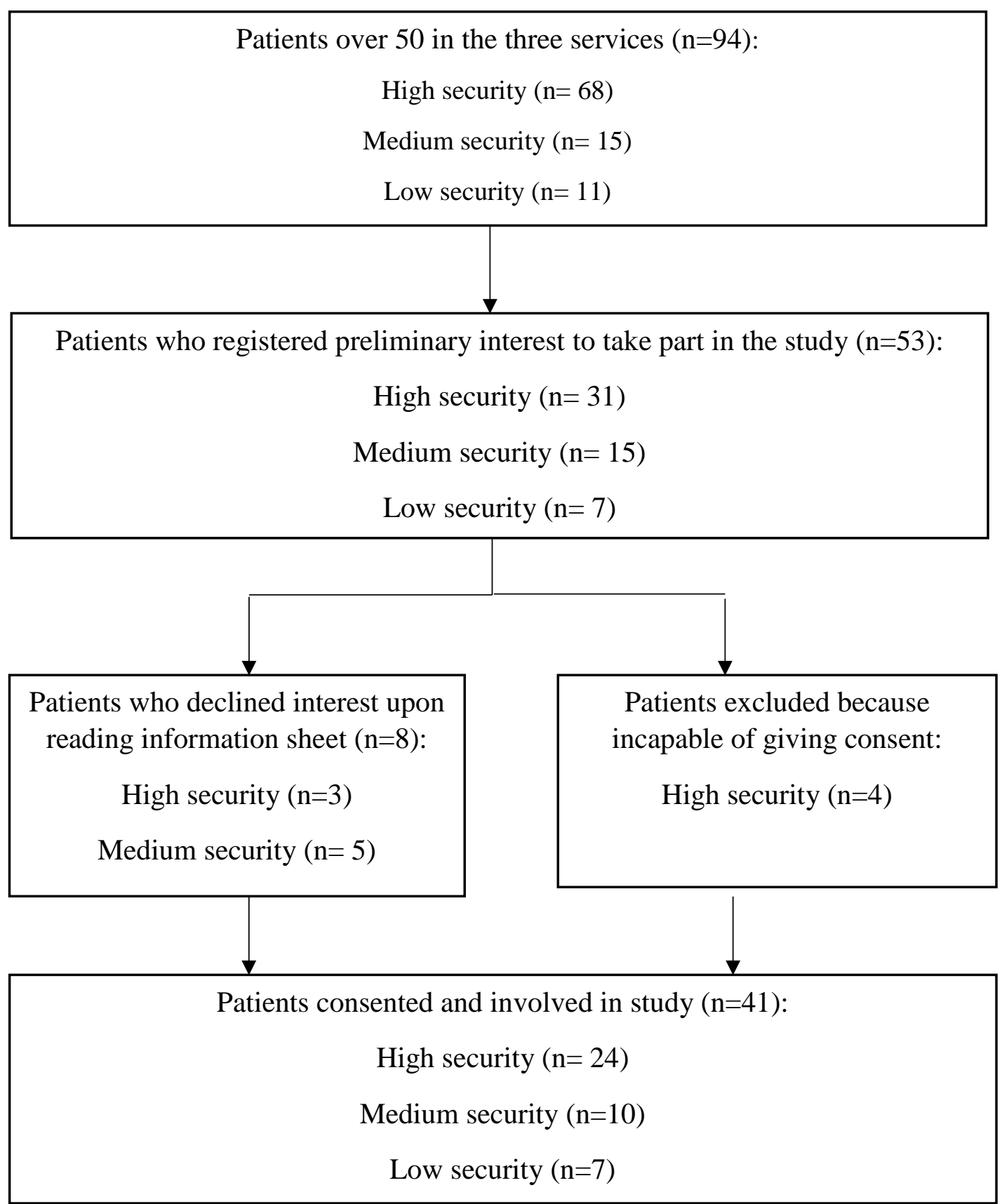


Table 1. Correlation and inferential analyses carried out in this study

\begin{tabular}{|c|c|c|}
\hline Type of statistics & Objective 1 & Objective 2 \\
\hline Chi-Square & & $\begin{array}{l}\text { To test differences between our sample and the overall population over } 50 \text { in the } \\
\text { services around: } \\
\text { - Gender } \\
\text { - Ethnicity } \\
\text { - Marital status } \\
\text { To test the association between participants' diagnoses and: } \\
\text { - Age } \\
\text { - Gender } \\
\text { - Ethnicity } \\
\text { To test the association between physical health conditions and: } \\
\text { - Gender } \\
\text { - Age } \\
\text { - Medications } \\
\text { To test the association between seclusion and psychiatric disorders }\end{array}$ \\
\hline Independent Samples T-test & & $\begin{array}{l}\text { To test the association between HCR-20 V3 total score and: } \\
\text { - Diagnosis } \\
\text { - Index offence } \\
\text { - Medications } \\
\text { To test the association between the presence of needs and: } \\
\text { - Gender } \\
\text { - Age }\end{array}$ \\
\hline
\end{tabular}




\begin{tabular}{|c|c|c|}
\hline & & $\begin{array}{l}\text { - Diagnosis } \\
\text { - Treatment } \\
\text { - Type of ward } \\
\text { To test the association between met needs and: } \\
\text { - Gender } \\
\text { - Age } \\
\text { - Diagnosis } \\
\text { - Treatment } \\
\text { - Type of ward } \\
\text { - Seclusion. } \\
\text { To investigate any statistically significant difference between CAMCOG-R scores } \\
\text { in our sample against the sample in Pereiro, Ramos-Lema, Juncos-Rabadán, Facal, } \\
\text { and Lojo-Seoane, } 2015\end{array}$ \\
\hline Mann-Whitney & $\begin{array}{l}\text { To test the association } \\
\text { between length of stay } \\
\text { and gender }\end{array}$ & $\begin{array}{l}\text { To test the association between length of stay and age } \\
\text { To test the association between medications and HCR-20 V } 3 \text { Clinical and History } \\
\text { scales. } \\
\text { To test the association between unmet needs and: } \\
\text { - Age } \\
\text { - Gender } \\
\text { - Diagnosis } \\
\text { - Treatment } \\
\text { - Type of ward } \\
\text { To test the association between CAMCOG-R score and: }\end{array}$ \\
\hline
\end{tabular}




\begin{tabular}{|c|c|c|}
\hline & & $\begin{array}{l}\text { - Gender } \\
\text { - Psychiatric diagnosis } \\
\text { - Medications }\end{array}$ \\
\hline $\begin{array}{l}\text { One way ANOVAs and } \\
\text { Tukey post hoc tests }\end{array}$ & & $\begin{array}{l}\text { To investigate any statistically significant difference between HCR-20 V3 scores in } \\
\text { our sample against the sample in Morissey, Beeley, and Milton, } 2014\end{array}$ \\
\hline Kruskal-Wallis & $\begin{array}{l}\text { To test the association } \\
\text { between type of service } \\
\text { and: } \\
\text { - Length of stay } \\
\text { - Age }\end{array}$ & To test the association between length of stay and type of service \\
\hline Correlation & & $\begin{array}{l}\text { To test correlation between CAMCOG-R score and: } \\
\text { - Length of stay } \\
\text { - Age } \\
\text { - HCR-20 V3 score } \\
\text { - PCL-R score } \\
\text { - Number of unmet needs }\end{array}$ \\
\hline
\end{tabular}


Table 2. Patients' sociodemographic data and data on residency (objective 1)

\begin{tabular}{r|lllr}
\multicolumn{2}{c}{} & $n$ & $\%$ & $\begin{array}{r}95 \% \text { CI } \\
(\%)\end{array}$ \\
\hline \multirow{5}{*}{ Age group } & $50-59$ & 73 & 77.7 & $68-84$ \\
& $60-69$ & 20 & 21.3 & $14-30$ \\
& $70+$ & 1 & 1 & $0-5$ \\
Ethnicity & Male & 84 & 89.4 & $81-94$ \\
& Female & 10 & 10.6 & $0-15$ \\
& White-British & 75 & 80 & $70-86$ \\
& White (other) & 5 & 5 & $2-12$ \\
& Mixed (any) & 2 & 2 & $0-7$ \\
& Black/Black-British & 10 & 11 & $5-18$ \\
& Asian/Asian-British & 2 & 2 & $0-7$ \\
Rarital status & Single & 74 & 78.8 & $69-85$ \\
& Divorced/separated & 18 & 19.2 & $12-28$ \\
& Married & 1 & 1 & $0-5$ \\
& Widowed & 1 & 1 & $0-5$ \\
& HS & 68 & 72 & $62-80$ \\
& MS & 15 & 16 & $9-25$ \\
& LS & 11 & 12 & $6-20$
\end{tabular}


Table 3. Patients' sociodemographic data, data on residency and index offence (objective 2)

\begin{tabular}{|c|c|c|c|c|}
\hline & & $n$ & $\%$ & $\begin{array}{r}95 \% \mathrm{Cl} \\
(\%)\end{array}$ \\
\hline \multirow[t]{3}{*}{ Age group } & $50-59$ & 32 & 48.8 & $33-64$ \\
\hline & $60-69$ & 8 & 19.5 & $7-32$ \\
\hline & $70+$ & 1 & 2.4 & $0-7$ \\
\hline \multirow[t]{2}{*}{ Gender } & Male & 38 & 92.7 & $85-100$ \\
\hline & Female & 3 & 7.3 & $0-15$ \\
\hline \multirow[t]{4}{*}{ Ethnicity } & White-British & 34 & 83 & 71-94 \\
\hline & White (other) & 3 & 7 & $0-15$ \\
\hline & Mixed (any) & 2 & 5 & $0-12$ \\
\hline & Black/Black-British & 2 & 5 & $0-12$ \\
\hline \multirow{4}{*}{ Marital status } & Single & 31 & 75.6 & $62-89$ \\
\hline & Divorced/separated & 7 & 17.1 & $5-29$ \\
\hline & Married & 2 & 4.9 & $0-11$ \\
\hline & Widowed & 1 & 2.4 & $0-11$ \\
\hline \multirow[t]{6}{*}{ Religion } & Christian & 22 & 54 & $39-69$ \\
\hline & Muslim & 2 & 4.8 & $0-11$ \\
\hline & Buddhist & 2 & 4.8 & $0-11$ \\
\hline & Other & 3 & 7.2 & $0-15$ \\
\hline & Atheist & 1 & 2.3 & $0-7$ \\
\hline & Undisclosed & 11 & 26.9 & $13-41$ \\
\hline \multirow[t]{3}{*}{ Residency } & $\mathrm{HS}$ & 24 & 58.5 & $43-73$ \\
\hline & MS & 10 & 24.4 & $11-38$ \\
\hline & LS & 7 & 17.1 & $5-29$ \\
\hline \multirow[t]{5}{*}{ Current ward } & Male mental health & 19 & 46.3 & $31-61$ \\
\hline & Personality disorders & 14 & 34.1 & $20-49$ \\
\hline & Male learning disability & 4 & 10 & $1-19$ \\
\hline & Women services & 3 & 7.3 & $0-15$ \\
\hline & Male community integration & 1 & 2.4 & $0-11$ \\
\hline \multirow[t]{8}{*}{ Index offence } & Assault* & 16 & 39 & $24-54$ \\
\hline & Manslaughter** & 9 & 22 & $9-35$ \\
\hline & Murder & 5 & 12.2 & $2-22$ \\
\hline & Sexual offence & 4 & 9.8 & $1-19$ \\
\hline & Other*** & 11 & 26.8 & $13-40$ \\
\hline & No index offence & 1 & 2.4 & $0-11$ \\
\hline & Multiple offences & 5 & 12.2 & $2-22$ \\
\hline & Previous index offence & 27 & 65.8 & $51-80$ \\
\hline
\end{tabular}

* Including common assault, battery, assault occasioning actual or grievous bodily harm ** Including on the grounds of diminished responsibility *** Including criminal damage, firearms, offensive weapons, bomb threats and hoaxes, theft and dishonesty, drug offences, immigration, evasion of liability and bail offences 
Table 4. Patients' psychiatric diagnoses, prescribed medications, treatment, physical condition and incidents (objective 2)

\begin{tabular}{|c|c|c|c|c|}
\hline & & $n$ & $\%$ & $\begin{array}{r}95 \% C I \\
(\%)\end{array}$ \\
\hline \multirow[t]{11}{*}{ Diagnosis } & Personality disorder & 24 & 60 & $45-75$ \\
\hline & Schizophrenia & 17 & 41.5 & $26-56$ \\
\hline & Schizoaffective disorder & 2 & 4.9 & $0-11$ \\
\hline & Other psychotic disorder & 1 & 2.4 & $0-7$ \\
\hline & Bipolar & 6 & 14.6 & $4-25$ \\
\hline & Depression & 3 & 7.3 & $0-15$ \\
\hline & Anxiety disorder (any) & 6 & 14.6 & $4-25$ \\
\hline & Substance misuse/dependence (any) & 6 & 14.6 & $4-25$ \\
\hline & Learning disability & 4 & 9.8 & $1-19$ \\
\hline & Dementia & 0 & 0 & - \\
\hline & Comorbid disorders (any) & 22 & 53.7 & $38-69$ \\
\hline \multirow[t]{6}{*}{ Medication } & Any psychotropic & 36 & 87.8 & $78-98$ \\
\hline & Antipsychotic & 34 & 82.9 & 71-94 \\
\hline & Mood stabiliser & 10 & 24.4 & $11-38$ \\
\hline & Antidepressant & 9 & 22.0 & $9-35$ \\
\hline & Benzodiazepine & 8 & 19.5 & $7-32$ \\
\hline & Other $^{+}$ & 3 & 7.3 & $0-15$ \\
\hline \multirow{9}{*}{ Treatment } & Skills development & 30 & 72.2 & $58-86$ \\
\hline & Mental health awareness/psychoeducation & 12 & 29.3 & $15-43$ \\
\hline & Art therapy & 8 & 19.5 & $7-32$ \\
\hline & Healthy life-styles training & 8 & 19.5 & $7-32$ \\
\hline & Dialectical behaviour therapy & 7 & 17.1 & $5-29$ \\
\hline & Substance misuse treatment & 7 & 17.1 & $5-29$ \\
\hline & Music/dance therapy & 6 & 14.6 & $4-25$ \\
\hline & Violence reduction & 6 & 14.6 & $4-25$ \\
\hline & Preparation for therapy/motivational work & 6 & 14.6 & $4-25$ \\
\hline \multirow{12}{*}{$\begin{array}{l}\text { Physical } \\
\text { condition }\end{array}$} & At least one documented condition & 36 & 87.8 & 78-98 \\
\hline & Diabetes & 11 & 26.8 & $12-41$ \\
\hline & Heart & 10 & 24.4 & $10-38$ \\
\hline & High blood pressure & 9 & 22.0 & $8-35$ \\
\hline & Obesity & 9 & 22.0 & $8-35$ \\
\hline & Gastrointestinal system & 9 & 22.0 & $8-35$ \\
\hline & Musculoskeletal system & 9 & 22.0 & $8-35$ \\
\hline & Respiratory & 6 & 14.6 & $3-26$ \\
\hline & Sensory impairment & 4 & 9.7 & $0-19$ \\
\hline & Epilepsy & 2 & 4.9 & $0-12$ \\
\hline & Cancer & 1 & 2.4 & $0-7$ \\
\hline & Any other & 13 & 31.7 & $16-47$ \\
\hline \multirow[t]{2}{*}{ Psychopathy } & Score available & 14 & 34 & $21-49$ \\
\hline & Score unavailable & 17 & 66 & $27-56$ \\
\hline
\end{tabular}


Score above national threshold for

psychopathy (>25)

Score below national threshold for psychopathy $(<25)$

+ Including barbiturates and stimulants (methylphenidate) $\begin{array}{lll}8 & 64.3 & 32-78\end{array}$

$5 \quad 35.7 \quad 16-61$ 